\title{
Practical Implementation of an Indoor Robot Localization and Identification System using an Array of Anchor Nodes
}

\author{
Israa Sabri A. AL-Forati*1, Abdulmuttalib T. Rashid ${ }^{2}$ \\ ${ }^{1}$ Electrical Engineering Department, University of Basrah, Basrah, Iraq \\ ${ }^{2}$ Electrical Engineering Department, University of Basrah, Basrah, Iraq
}

\author{
Correspondence \\ * Israa Sabri A. AL-Forati \\ Almanawe, Basrah, Iraq. \\ israa.subri.1@gmail.com
}

\begin{abstract}
This paper proposes a low-cost Light Emitting Diodes (LED) system with a novel arrangement that allows an indoor multirobot localization. The proposed system uses only a matrix of low-cost LED installed uniformly on the ground of an environment and low-cost Light Dependent Resistor (LDR), each equipped on bottom of the robot for detection. The matrix of LEDs which are driven by a modified binary search algorithm are used as active beacons. The robot localizes itself based on the signals it receives from a group of neighbor LEDs. The minimum bounded circle algorithm is used to draw a virtual circle from the information collected from the neighbor LEDs and the center of this circle represents the robot's location. The propose system is practically implemented on an environment with (16*16) matrix of LEDs. The experimental results show good performance in the localization process.
\end{abstract}

KEYWORDS: Multi-robot, Localization, Binary search algorithm, Minimum bounded circle algorithm.

\section{INTRODUCTION}

In general term, localization can be defined as a process of determining the position of object in some environment. The Localization is an important issue in multi-node or multirobot systems. The multi-robot positioning property offers new occasions in many fields such as: path planning and obstacle avoidance, object tracking, inspection, surveillance and multi-mobile robot formation [1]. In recent years, the localization of mobile robots has proved a difficult task due to many physical limitations. In the mobile robotics field, the robot localization systems and formation have been of particular interest [2]. In these scenarios, robots may be required to navigate based on information or to improve their path planning [3]. The most famous localization technique is the global positioning system (GPS), but constraints such as its high cost, power consumption and large size make this method difficult to use regularly [4]. In addition, for indoor environments GPS cannot be used; this fact has attracted growing attention to proposing more algorithms that deal with the needs of indoor localization [1,5]. A Network of Wireless Sensors consists of multiple nodes of sensors divided separately to cooperatively monitor environmental circumstances and has different applications such as traffic monitoring, object tracking, navigation of ships, and measuring radiation levels from nuclear reactors, and monitoring volcanic eruptions [6]. A sensor is a small instrument that is used to measure or sense roughly natural quantity and then translate it via some relationship to a new motion that can then be read by people for display or for further processing. Also, they are used for diverse kinds of measurement, for example: distance, sound, motion, pressure, temperature, light levels. [7, 8]. With the rapid increase in smart technology, we are looking for a sensor that provides precise distance detection; also, it should be low in cost. The accuracy and inexpensiveness of light emitting diode (LED) sensors, along with their extended life expectancy, is valuable: lights are expected to continue for many years; moreover, the light is harmless to humans, unlike IR and lasers, and it does not overlap with the effects of electronic devices in the same environment. The foregoing features makes LEDs a very suitable choice for use in localization scenarios. Practical experiments can test the light sensors with low-cost LEDs \& LDRs.

Several procedures are used for indoor localization such as the systems built on ultra-wideband, Wi-Fi, radio frequency 
identification (RFID), Bluetooth, ultrasound, and infrared [9, 10]. Indoor localization using Bluetooth Low Energy (BLE) beacons is widely used after the appearance of the BLE protocol. Bluetooth beacons have two roles: broadcasting by beacon and receiving by sensing devices. Thus, many researchers are implementing positioning methods that use beacons [11]. One of the researchers uses the combination of channel-separate polynomial regression model (PRM), channel-separate fingerprinting (FP), outlier detection and extended Kalman filtering (EKF) for indoor localization using smartphone with BLE beacons. This localization algorithm uses FP and PRM to estimate the target's location [12]. Another researcher describes basic principles of a radio-based indoor localization and focuses on the improvement of its results with the aid of a new Bluetooth Low Energy technology. His system enables volunteers to create radio-maps and update them continuously. In this system BLE transmitters have been installed on the ground of the building in addition to present $\mathrm{Wi}-\mathrm{Fi}$ access points. [13]. In all these technologies used in positioning systems, the complexity changes significantly the price and precision. All the localization systems mentioned above fall into two categories: the first uses only one photodiode (LDR) in the receiver; the second employs more than one, such as the receiver presented in [14] that employs a lens and an image sensor and computes the path of arrival of light collected from diverse visible LEDs. In the proposed new system, there is an advantage of lower hardware complexity but with more accurate and realistic results, along with high speed to find the required position. Within the first category, some receivers, such as those reported in $[15,16]$, rely on the received signal strength to calculate their distance from the beacons, while others, such as those in $[17,18]$, attempt to use the difference in the propagation delays from diverse beacons. Synchronization through the transmitter is an essential element in all the solutions cited, and this is expensive for implementation, while the proposed system does not need any synchronization. The new algorithm is a binary procedure, returning a number of available node receivers in the environment, then approximating the result using minimum bounded circle algorithm to include all these nodes, then the center of this circle is assumed to be the robot's position. In this paper, a new-fangled localization algorithm is planned to overcome all the restrictions in implementation of the above systems. In this algorithm, the localization is based on designing a system with a pair of sensors: the first (group of LDRs) is fixed on the robot as receiver sensor and the other (matrix of white LEDs) works as the transmitter sensor, distributed uniformly throughout the system. For a number of receivers (LDRs), the minimum bounded circle algorithm is used to draw a minimum circle that contains all of the points; as a result, the center of this circle is assumed to be the robot's position.

\section{Practical ImPlementation OF The Robot LOCALIZATION SYSTEM}

This section describes the practical implementation of the indoor robot localization system by scanning the array of LEDs in the environment. The localization is achieved using pairs of sensors: LDR sensor fixed on the robot represents the first part and the second is represented by matrix of white LEDs distributed uniformly in the environment. The binary search algorithm [19] is used to control the lighting of the matrix of LEDs and the data collected from the LDR sensors is directed to the central unit. The robot location is estimated by applying the minimum bounded circle algorithm [20] to the collected information. The procedure used to estimate the location of each robot in multi-robot system is similar to the procedure mentioned above when each robot has a different ID for communicating and sending the collected data to the main control unit. The problem occurs when the main control unit has not the intelligence to recognize the source of information received. In order to solve this problem and estimate the multi-robot locations, the k-means clustering algorithm is used to isolate the received information into clusters [21, 22]. The center of each cluster represents the location of one robot when using the proposed method.

The robot is represented by static node that sense the light of LEDs by a single LDR sensor. The diagram of the data collection infrastructure is shown in Figure 1. There are four main components in this system: the node localization environment, the node, the computer transmitter/receiver board and the data logging software. The environment has a matrix of LEDs and the coordinate axis of each LED is determined according to it is raw and column location in the LEDs array. During the scan process, the LDR sensor collect it is reading and send the information to the computer transmitter/receiver board. The localization system uses an NRF24L01 transmitter/receiver unit to wirelessly transfer data between the computer in one side and the node on the other side. The data logging software on the computer estimates the node location dependent on the reading of the LDR sensor and displays the estimated node location on the real-time GUI.

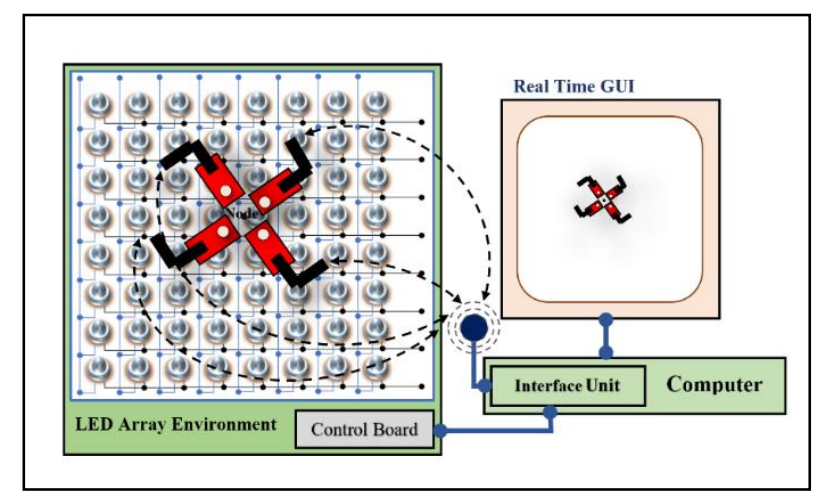

Fig. 1: Scheme of the nodes localization system.

\section{A. The node localization environment}

This environment is designed to work as a node localization system by using two-dimensional LEDs array as shown in Fig. 2. These LEDs are arranged in rows and columns structure and covered by a thin transparent layer of plastic. The anodes of each row in LEDs array are connected together to one output of the control unit to turn ON and OFF the LEDs in that row. The cathodes of each column in LEDs 
array are connected together to one output of a decoder circuit to turn ON and OFF the LEDs by scan these columns. The decoder circuit is controlled by some outputs of the main control unit. The turn ON and turn OFF the LEDs depends on using the binary search algorithm to minimize the required time for scanning the rows and the columns of the array of LEDs [21]. Each LED coordinate axis depends on its row and column locations in the LEDs array. The main control part of the localization system receives the orders from PC to turning ON and OFF the LEDs using the binary search algorithm. At each order from the PC, the main control part uses the collected information from the LDR sensors to localize the node. Fig. 3 shows the schematic diagram of the main control board.

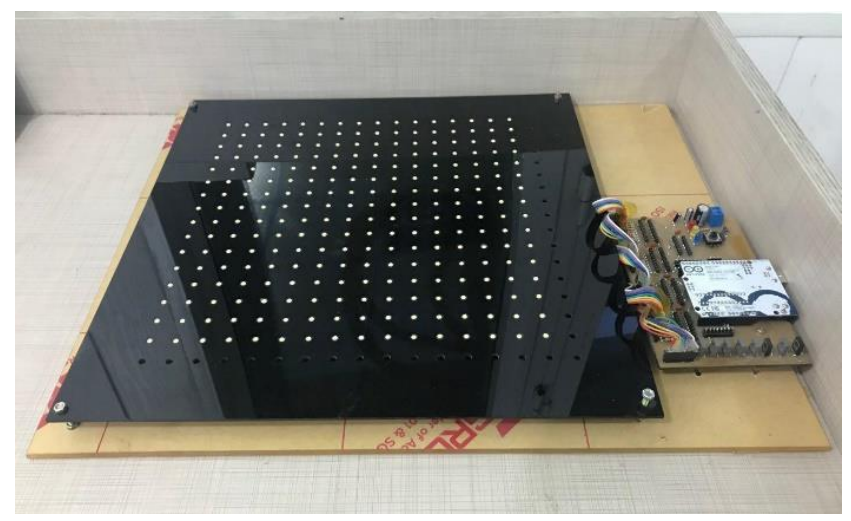

Fig. 2: The robot localization environment.

Four 74HC574N D Flip Flops are used in this unit, each one is used to latch on byte in each quarter of the LEDs array. A scan principle is used to make all the LEDs appears as latch at the same time. The scanning principle is implemented by using one $74 \mathrm{HC} 138 \mathrm{~N}$ decoder and eight BD137 NPN transistors. An Arduino UNO microcontroller is used to control the operating of the four D F/F and the decoder circuit using the binary search algorithm. The collected information from the nodes LDR sensors are transfer wirelessly to the Arduino UNO microcontroller unit which connect to the PC using NRF Transmitter/Receiver module.

\section{B. The node designs}

The node localization process is achieved by sending the node information which collected from the environment to the main control unit. The localization process is achieved by analysis the collected information using the minimum bounded circle algorithm [22]. The node is a small rectangular board (Fig. 4) equipped by Arduino NANO microcontroller, LDR sensor, NRF Transmitter/Receiver module and battery. Figure 5 shows the schematic diagram of the robot main control part. The microcontroller in this board receives the orders from the PC to sense the environment when the LED array is scanned. The node gives the orders to the LDR sensors to sense the status of the LED arrays and transfer the reading data wirelessly (using the NRF24L01 board) to the Computer transmitter/receiver board.

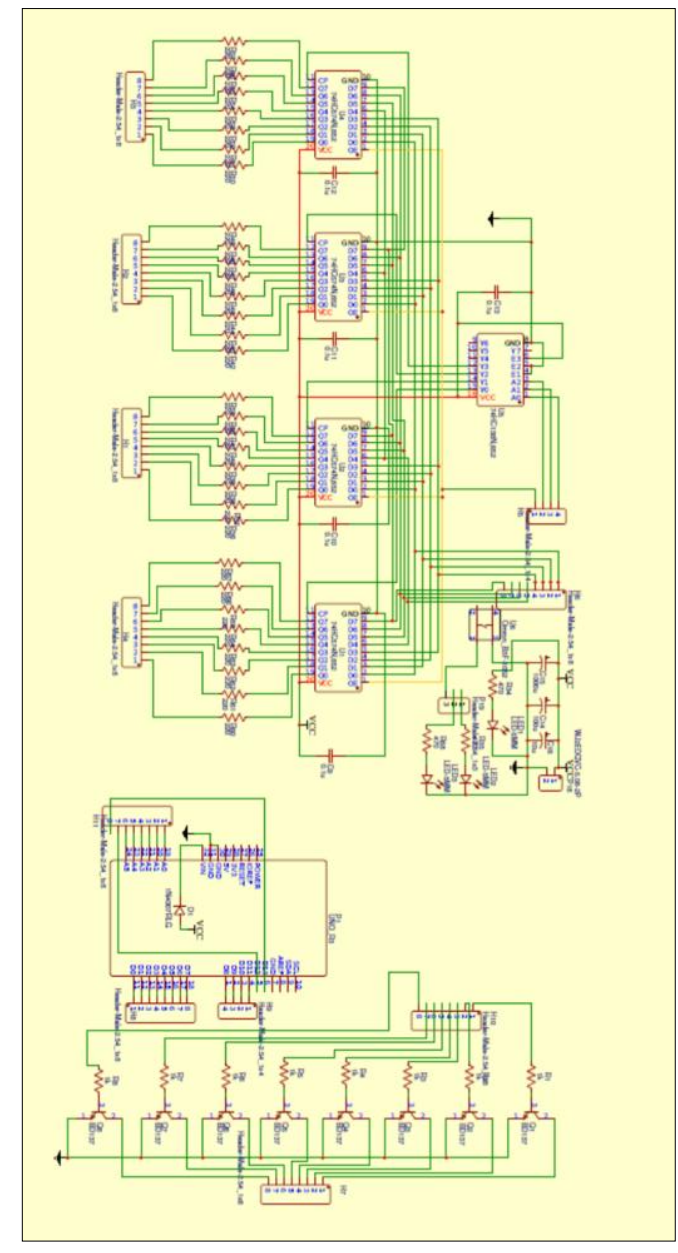

Fig. 3: Schematic diagram of the latch and driver circuits for the main control board.

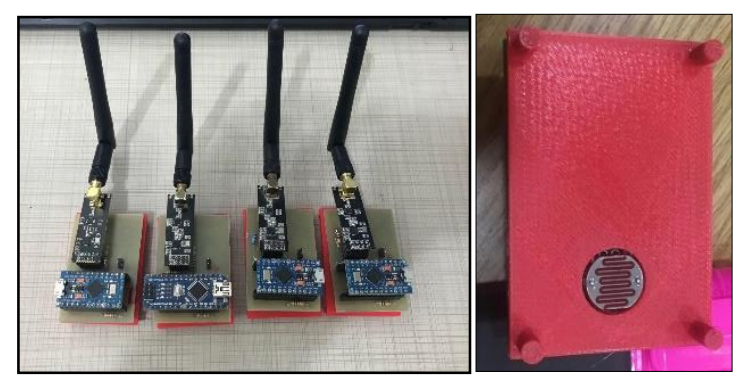

(a)

(b)

Fig. 4: Multi-Nodes. (a) Four nodes (b) The lower side of node.

\section{The computer transmitter/receiver board}

The computer trans-receiver board is designed to transmit and received the information wirelessly between the computer and the nodes. This board equipped by Arduino "pro micro" microcontroller and NRF Transmitter/Receiver module. The microcontroller is programmed to transmit the order to the nodes to start sensing the environment by the LDR sensor. This process keeps the synchronization between the time of applying the binary search algorithm by the computer and the suitable time to sense the status of the 
LEDs array by the nodes. After that the computer transreceiver board wait to receive the sensing data from the nodes and transfer it to the computer. The computer manipulates this data to estimate the current location of the nodes. Fig.6. (a) and (b). show the computer trans-receiver board and it is printed circuit board (PCB).

\section{Data logging software}

The sub section describes the software part of the multi-node localization system. Data logging software is designed to estimate the nodes locations using the binary search algorithm. It is localization process dependent on the data that collect from the nodes and the localization environment. The Graphic User Interface (GUI) for this software (Fig. 7) has two parts: LDR information and Error estimation. The LDR information part is used to select the actual location of node ( $\mathrm{x}, \mathrm{y})$ coordinate axis), number, diameter and sensing range of the LDR sensor. The Error estimation part is used to display the actual position, estimated position and error estimation for node localization. Data logging software represents by four programs: the main computer program, the trans-receiver microcontroller program, the localization system program and the node microcontroller program.

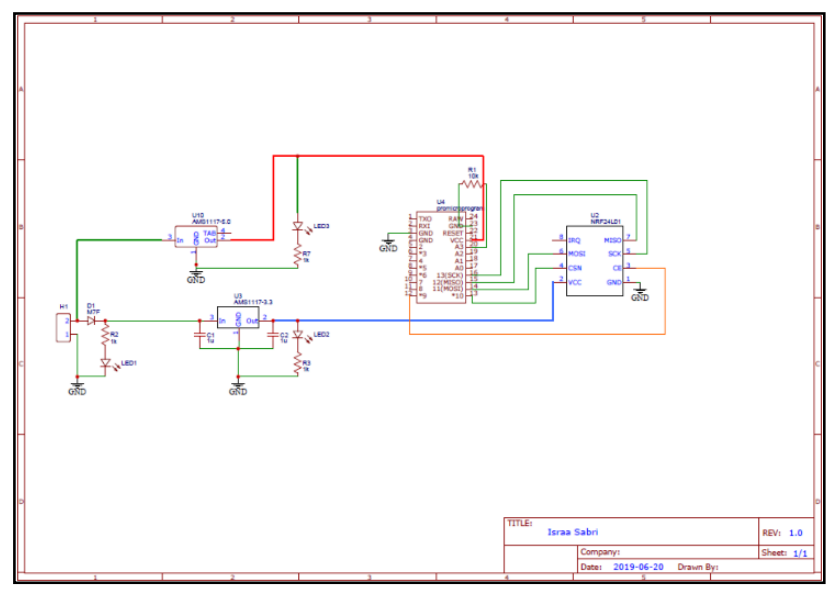

Fig. 5: Schematic diagram for the node.

\section{THE MAIN COMPUTER PROGRAM}

This program is written using Visual Basic Language to control the turn on and turn of the LEDs of the localization environment according to the binary search algorithm and also to collect the sensing data from the nodes which used in nodes localization and error estimation according to the minimum bounded circle algorithm. The localization process is started be gave the orders to the localization system to lighting the LEDs. The main control unit wait to receive the LDR sensor information collected by the nodes. The main program used the collected information to draw the distribution of the nodes on the GUI. Fig. 8 shows the flowchart to the main control unit.

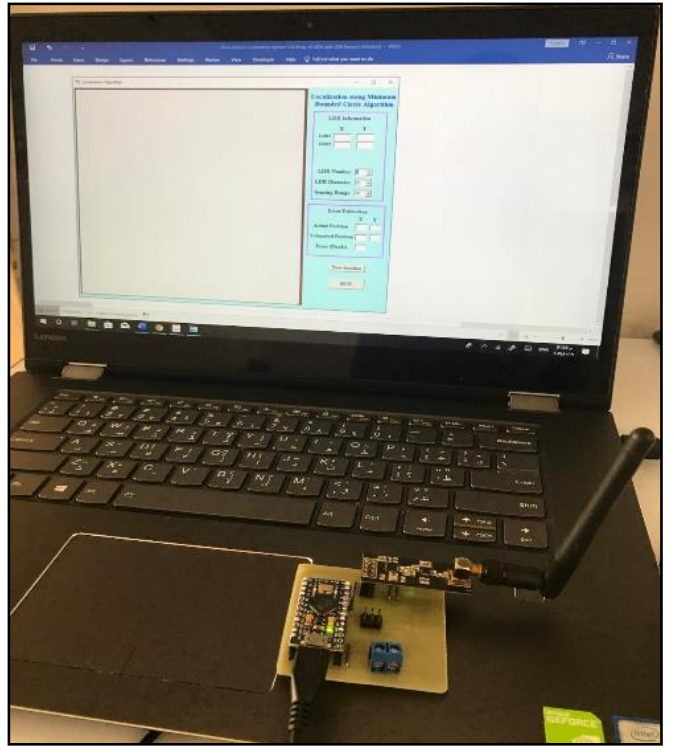

(a)

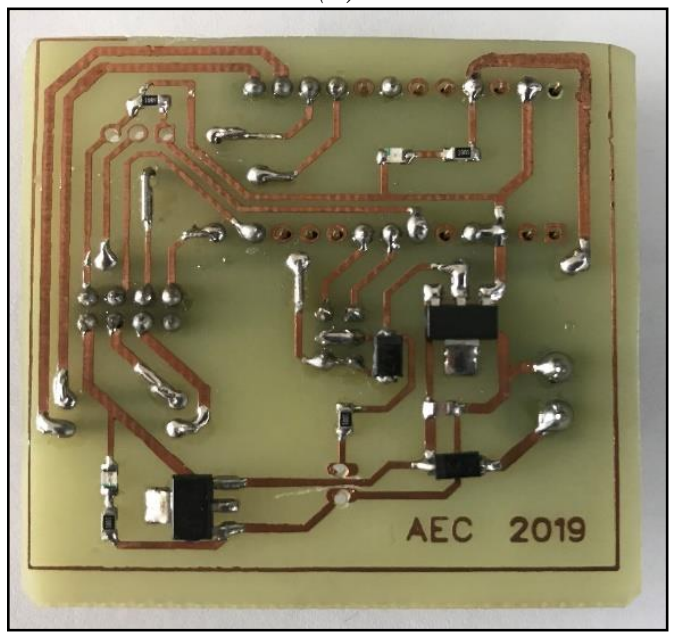

(b)

Fig. 6: The trans-receiver boards. (a) The computer transreceiver board. (b) The printed circuit board (PCB) of the computer trans-receiver board.

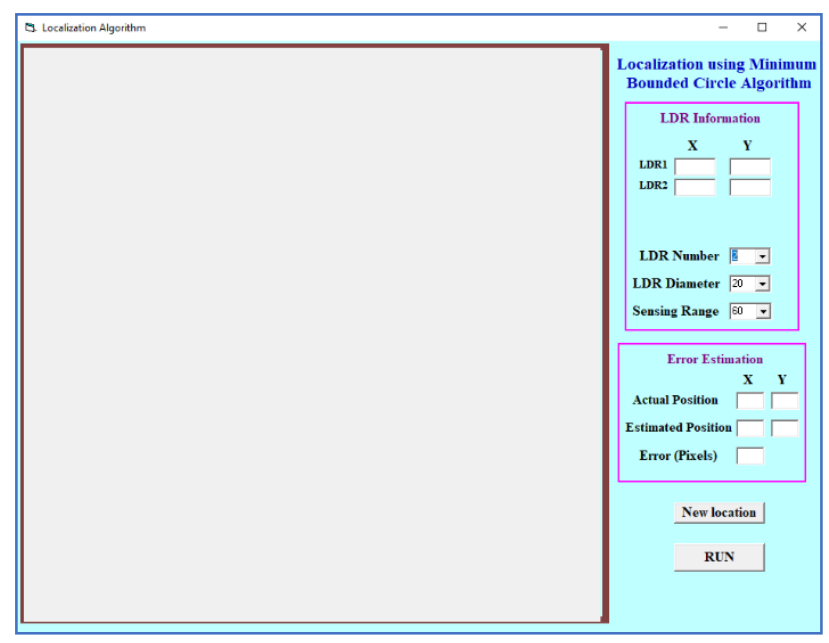

Fig. 7: The GUI for the software of the node localization system. 


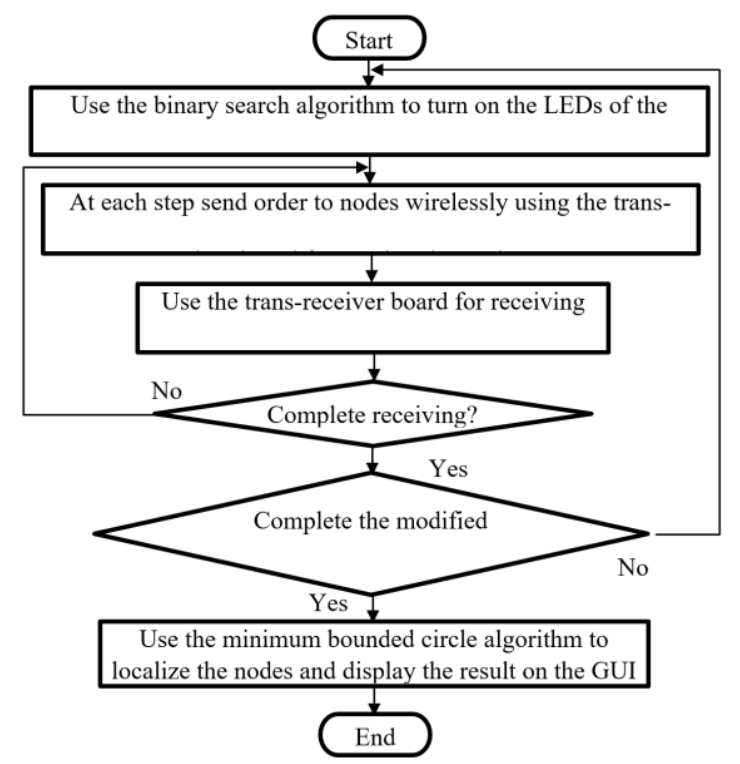

Fig. 8: Flowchart for the main program.

\section{A. The trans-receiver microcontroller program}

The computer is connected to the microcontroller of the trans-receiver unit, which used to communicate wirelessly to the nodes. The program used on this board is to receive the orders from the computer and transfer this order wirelessly to the nodes and also to receive the sensing information from nodes and transfer them to the computer.

\section{B. The trans-receiver microcontroller program}

This program is C-language program store in the microcontroller of the localization system board. According to the binary search algorithm, this program received the orders from the main program to turn on and turn off the LEDs using the scan principle. The scan principle is used to distribute the D flip flops information among the columns of the LEDS matrix by using a decoder circuit. This process of LEDs lighting is repeated also for the rows of the same LEDs matrix. Fig. 9 shows the flowchart of the localization system program.

\section{The The node program}

This is a C-language program store on the node microcontroller. Each node has RF module and one LDR sensor. It receives the orders from the trans-receiver board to start sensing the LEDs information. The collected data is sent wirelessly to the trans-receiver board. Fig. 10 shows the flowchart of the node program.

\section{EXPERIMENTAL RESULTS}

In this paper, an original robotic localization scheme is validated by experimental. Experimental are performed for the single and multi-robot system with single of LDR sensor. The experiments are repeated for 50 different topologies representing different robot locations and different sensing ranges for the LDR sensors. The system parameters used in these experiments are:

1) Maximum sensing range of the LDR sensor.

2) Number of LEDs in the LED matrix.

The experimental results are implemented to show the performance of single and multi-node localization system using $(16 \times 16)$ LED matrix environment. The distances among any two LEDs in this environment are $2 \mathrm{Cm}$. The LEDs lighting are controlled by Arduino microcontroller and four D flip flops which receive the orders from the main computer. In simulation we found that the best performance in localization process is obtained when using robot with single LDR sensor, so that in this section all the experiments are implemented on nodes with single LDR sensor. The first group of localization experiments are implemented on environment with single node as shown in Fig. 11. Two experiments are tested in this group: The average of detecting neighbor LEDs and the average of the errors in the localization process. At first experiment, different values for LDR sensor range is tested and at each value the experiment is repeated for 20 times to compute the average number of the detected neighbor LEDs. Fig. 12 shows the relationship among the LDR sensing range and the average of the detected neighbor LEDs. From Figure we found that as the sensing range of the LDR increase also the average of the detecting neighbor LEDs increase. In the second experiment, the average of node localization error is tested with respect to the sensing range of the LDR sensor. At each value of the sensing range the experiment is repeated for 20 times to compute the average of the error.

Fig. 13 shows the relationship among the sensing range of the LDR sensor and the average of the error in the localization process. This relationship shows that as the sensing range of the LDR sensor increase the average of the error in the localization process also increases.

The second group of localization experiments are implemented on environment with four nodes as shown in Fig. 14. This experiment is used to show the relationship among the average of the iterations of the k-mean clustering and the sensing range of the LDR sensors as shown in Fig. 15.

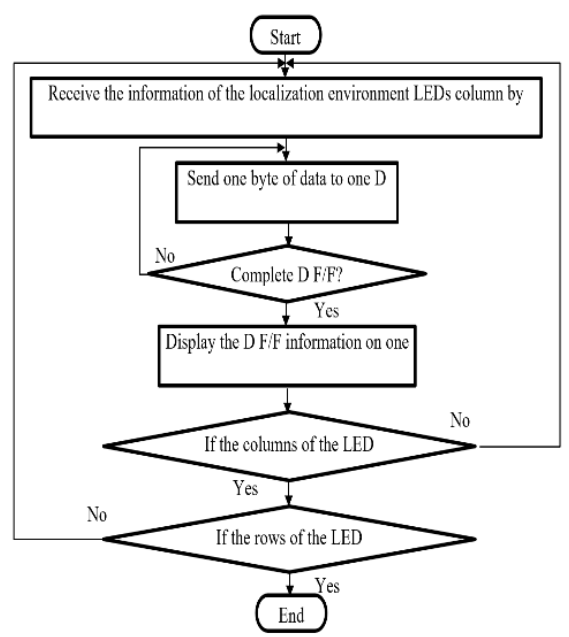

Fig. 9: Flowchart for localization system program. 


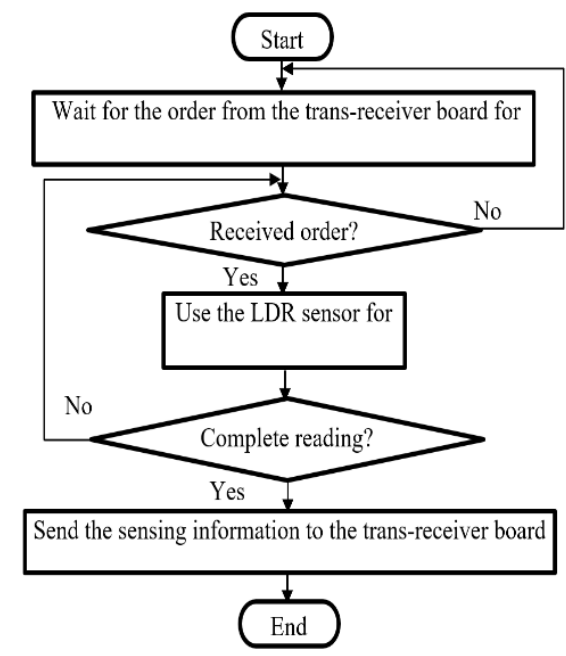

Fig. 10: Flowchart for robot program.

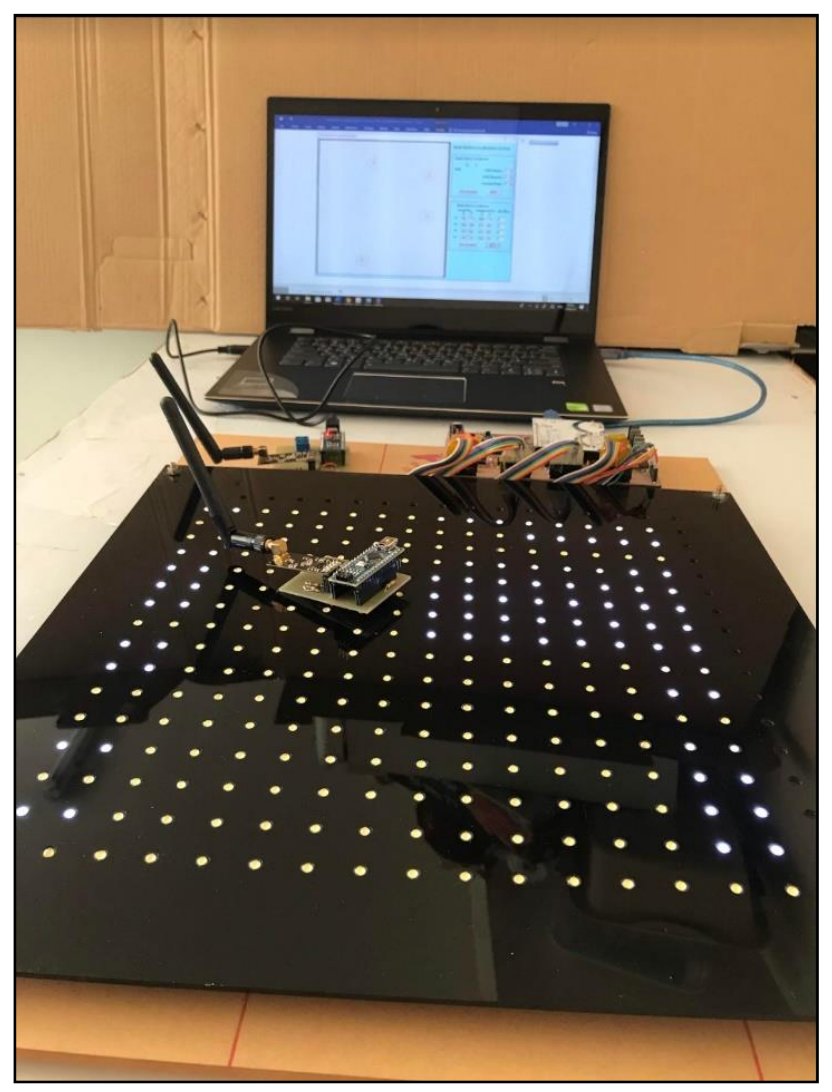

Fig. 11: Practical node localization environment.

By repeating this experiment 20 times, the result shows that as the sensing range of the LDR sensor is increased, the average repeated iteration numbers also increase.

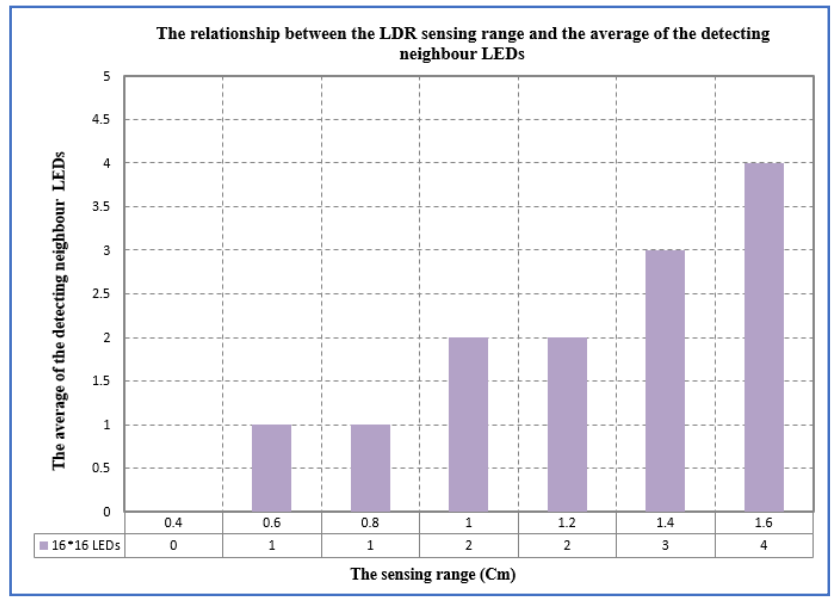

Fig. 12: The Comparison between the average of the neighbor LEDs and the LDR sensing range in $(16 \times 16)$ LED matrix environment.

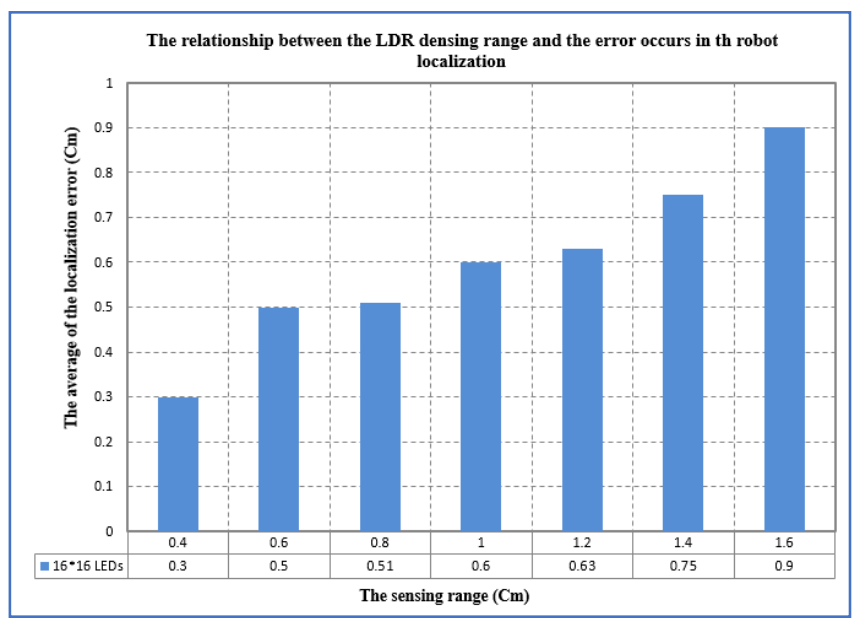

Fig. 13: The relationship among the average of the error in robot localization and the LDR sensing range in $(16 \times 16)$ LED matrix environment.

\section{Conclusion}

An indoor robot localization system is introduced. This system solves the problem of localization by using an array of LEDs distributed uniformly in the environment. The localization process is practically implemented and tested on single robot and multi-robot system with different sensing ranges. In practical design, the environment has $(16 \times 16)$ LED matrix and the node equipped with one LDR sensor. The experimental results show that as the sensing range of the LDR sensor is increased the average of detecting neighbor LEDs are also, increased which lead to increase the average of the error in the localization process. Also, in the experimental results of the multi-node localization environment, as the sensing range is increases, the average repeated iteration numbers for the K-means clustering algorithm also increases. 


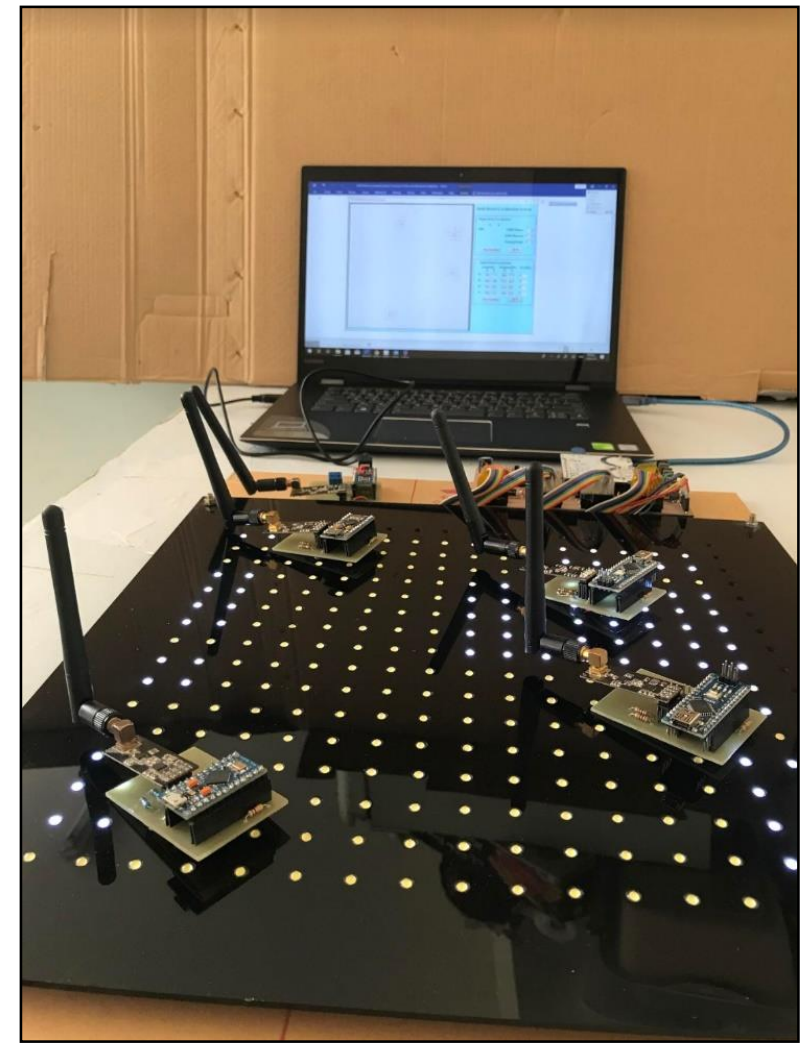

Fig. 14: The practical multi-node localization environment. The practical multi-node localization environment.

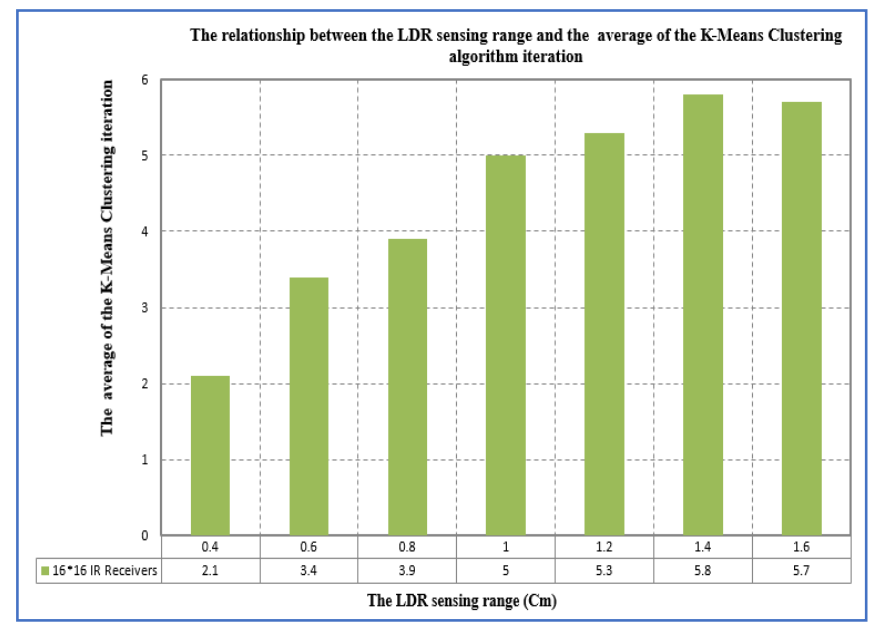

Fig. 15: The average iteration of the k-means clustering algorithm in robot localization with different LDR sensing range.

\section{CONFLICT OF INTEREST}

The authors have no conflict of relevant interest to this article.

\section{REFERENCES}

[1] A. T. Rashid, M. Frasca, A, A, Ali, A. Rizzo and L. Fortuna "Multi-robot localization and orientation estimation using robotic cluster matching algorithm,"
Robotics and Autonomous Systems, vol. 63, pp. 108121, 2015.

[2] R. Tron, J. Thomas, G. Loianno, K. Daniilidis and V. Kumar, "A Distributed Optimization Framework for Localization and Formation Control," Applications to Vision-Based Measurements. IEEE Contr. Sys. Mag., vol. 364, pp. 22-44, 2016.

[3] A.T. Rashid, A. A. Ali, M. Frasca, and L. Fortuna, "Path planning with obstacle avoidance based on the visibility binary tree algorithm," Robotics and Autonomous Systems, vol. 61, no. 12, pp. 1440-1449, 2013.

[4] N. B. Priyantha, H. Balakrishnan, E. Demaine, and S. Teller, "Anchor Free Distributed Localization in Sensor Networks," Tech Report \#892, 2003.

[5] A. T. Rashid, W. H. Zayer and M. T. Rashid, "Design and Implementation of Locations Matching Algorithm for Multi-Object Recognition and Localization," Iraqi Journal of Electrical and Electronic Engineering, vol. 14, no. 1, pp. 10-21, 2018.

[6] T. Arampatzis, J. Lygeros and S. Manesis, "A Survey of Applications of Wireless Sensors and Wireless Sensor Networks," Intelligent control. Proceedings of the 2005 IEEE International Symposium on, Mediterranean Conference on Control and Automation, pp. 719 - 724, 2005.

[7] O. A. Hasan, A. T. Rashid and R. S. Ali, "Centralized approach for multi-node localization and identification." Iraq J. Electrical and Electronic Engineering, vol. 12, no. 2, pp. 178-187, 2016.

[8] O. A. Hasan, A. T. Rashid, R. S. Ali, J. Kosha, "A practical performance analysis of low-cost sensors for indoor localization of multi-node systems", International Conference on Internet Technologies \& Applications, pp. 284-287, 2017.

[9] H. Liu, H. Darabi, P. Banerjee, and J. Liu, "Survey of Wireless Indoor Positioning Techniques and Systems". IEEE transactions on systems man, and cybernetics-part $C$ : application and reviews, vol. 37, no. 6, pp. $1067-$ 1080, 2007.

[10]I. S. Alfurati and Abdulmuttalib T. Rashid, "Performance Comparison of Three Types of Sensor Matrices for Indoor Multi-Robot Localization," International Journal of Computer Applications (09758887), vol.181, Issue 26, pp. 22-29, 2018

[11] Hyunwook Park, Jaewon Noh and Sunghyun Cho. "Three-dimensional positioning system using Bluetooth low-energy beacons," International Journal of Distributed Sensor Networks, vol. 12, no. 10, pp. 1-11, 2016.

[12] Yuan Zhuang, Jun Yang, You Li, Longning Qi and Naser El-Sheimy, "Smartphone-Based Indoor Localization with Bluetooth Low Energy Beacons," Sensors, vol. 16, pp. 1-20, 2016.

[13] Pavel Kriz, FilipMaly, and Tomas Kozel, "Improving Indoor Localization Using Bluetooth Low Energy Beacons," Mobile Information System, pp. 1-16, 2016.

[14] M. Yoshino, S. Haruyama, and M. Nakagawa, "Highaccuracy Positioning System using Visible LED Lights and Image Sensor," IEEE Radio and Wireless Symposium, 2008. DOI: 10.1109/RWS.2008.4463523. 
[15] S. Hann, J. H. Kim, S. Y. Jung and C. S. Park, "White LED ceiling lights positioning systems for optical wireless indoor applications," IEEE in 36th European Conference and Exhibition on Optical Communication, pp. 1-3, September 2010.

[16] Z. Zhou, M. Kavehrad and P. Deng (2012). "Indoor positioning algorithm using light-emitting diode visible light communications," Optical Engineering, vol. 51, no. 8, 2012.

[17] K. Panta and J. Armstrong (2012). "Indoor localization using White LEDs," ELECTRONICS LETTERS, vol. 48, no. 4, 2012.

[18] S. Jung, S. Hann and C. Park, "TDOA-Based Optical Wireless Indoor Localization Using LED Ceiling Lamps," IEEE Transactions on Consumer Electronics, vol. 57, no. 4, 2011

[19] E. E. Odokuma and O. O. Owolabi, “An Indexed Method for Improving the Efficiency of the Binary Search Algorithm," International Journal of Advanced Research in Computer Science and Software Engineering, vol. 6, no. 5. 2016.

[20] I. S. Alfurati and Abdulmuttalib T. Rashid, "Design and Implementation an Indoor Robot Localization System Using Minimum Bounded Circle Algorithm", The 8th International Conference on Modeling, Simulation and Applied Optimization (ICMSAO'2019), 2019

[21] L. Morissette and S. Chartier, "The k-means clustering technique: General considerations and implementation in Mathematica," Tutorials in Quantitative Methods for Psychology, vol. 9, no.1, pp. 15-24, 2013.

[22] I. S. Alfurati, A. T. Rashid and Alaa Al-Ibadi, "IR sensors array for robot's localization using $\mathrm{K}$ means clustering algorithm," International Journal of Simulation Systems, Science \& Technology, 12.1-12.6. 2019. 